\title{
LA CONSTRUCCIÓN DE DESIGUALDADES EN EL MERCADO DE TRABAJO DE LOS INVESTIGADORES EN MÉXICO
}

\author{
Guillermo Campos Ríos* \\ GERMÁn SÁNCHEZ DAZA** \\ María Eugenia Martínez de ITA***
}

Recebido: jun. 2010

Aprovado: jul. 2010

\begin{abstract}
*Profesor-Investigador de la Facultad de Economía de la BUAP. Coordinador de la investigación sobre el modelo de investigación predominante en los estados del centro de México (UAM/BUAP 2008-2009), proyecto colectivo en el que participaron los tres autores y que fue apoyado por la Vicerrectoría de Investigación y Estudios de Posgrado de la BUAP. E-mail: gcampos61@hotmail,com

** Profesor-Investigador de la Facultad de Economía de la BUAP. E-mail: sdaza@siu.buap.mx

*** Profesora-Investigadora de la Facultad de Economía de la BUAP. E-mail: cs000333@siu.buap.mx
\end{abstract}

Resumen: Este artículo muestra como un segmento de mercado como el de los investigadores en México - caracterizado por su alto nivel de educación- también se rige ciegamente por estructuras institucionales que finalmente reproducen inequidades similares a las que se encontrarían en cualquier otro segmento de trabajadores con menor nivel de educación; aunque, evidentemente la intensidad de las desigualdades es menor y en ocasiones aparecen metamorfoseadas. Este segmento está en una dinámica de crecimiento que avanza en sentido contrario a lo que ocurre en el actual contexto de destrucción masiva del empleo. Las desigualdades manifiestas están orientadas hacia las investigadoras y los jóvenes investigadores. A pesar de que en este mercado se mantienen condiciones laborales excepcionales respecto a otros segmentos, éstas son extraordinariamente frágiles. El principal obstáculo al crecimiento absoluto y racional de la actividad de investigación en México - dejando de lado la persistentemente baja inversión en I+D del gobierno mexicanoes la concentración de la investigación en el D.F. y en tres instituciones: la UNAM, La UAM y el Politécnico Nacional (IPN). Se concluye con la necesidad de pasar a una segunda fase de institucionalización de la investigación en México.

Palabra claves: Mercado de trabajo. Investigadores. Desigualdad investigadores.

\section{THE CONSTRUCTION OF INEQUALITY IN THE JOB MARKET OF THE RESEARCHERS IN MEXICO}

Abstract: This article shows how the labor market of researchers in Mexico - characterized by a high level of education - is also ruled by blind institutional structures that, finally, reproduce inequities in a similar way to those which we would encounter in any other workers' segment with a lesser level of education. Though, evidently, the intensity of inequities is smaller and in some occasions they turn out to be transfigured. This segment has a growth dynamic that advances in the opposite direction to what happens in the current context of the massive destruction of employment. Inequities are orientated towards female and young researchers and, although we can find exceptional working conditions with regard to other segments, these conditions are extraordinarily fragile. The main obstacle to the absolute and rational growth of the activity of research in Mexico - leaving the persistently low investment in $\mathrm{R}+\mathrm{D}$ of the Mexican government - is the concentration of the research in the F.D. and in three institutions: the UNAM, The UAM and the Politechnical Institute (IPN). We conclude with the need to go on to the second phase of institutionalization of research in Mexico.

Key words: Labor market; Researchers; Inequalities among researchers. 
Se habla más de los mercados de trabajo de lo que se ven, pues sus dimensiones las suelen fijar las ideas desconocidas y, quizás, místicas, de la mente de las personas [...] (CLARK KERR, 1954).

\section{INTRODUCCIÓN}

Al conocimiento -o la educación escolar como una de sus formas transfiguradas- se le ha concebido tradicionalmente como una herramienta útil para eliminar las desigualdades sociales. En los hechos, esta afirmación o no se cumple, o se cumple solo de forma parcial. Por ello se pensó que era adecuado estudiar al segmento laboral que dispone de la mayor cantidad de estudios escolares en este país: los investigadores, profesionales dedicados al cultivo de la ciencia, a efecto de conocer si en ese segmento social se eliminan definitivamente todas las diferencias que aparecen en otros segmentos con menor educación.

Los resultados muestran que hay una reproducción de desigualdades en el mercado de trabajo de los investigadores, con lo cual queda en entredicho la afirmación de que el conocimiento, en cuanto fuente de productividad marginal en los individuos- es una especie de antídoto de mercado contra la desigualdad. En esta investigación se encontró que este mercado se comporta -a otro nivel- con patrones de inequidad similares a los de cualquier otro mercado. Los términos de la desigualdad cambian y se metamorfosean pero subsisten.

Este artículo está sustentado por un lado en información ya construida, proveniente del CONACYT, pero hubo otra que se captó de fuente directa acudiendo con los investigadores a sus propios centros de investigación. La primera, corresponde a los investigadores registrados por el Sistema Nacional de Investigadores (SNI) para el año 2008, y algunos ajustes para el 2009, en todos los niveles de reconocimiento que el propio sistema considera (niveles I, II III y candidatos a investigador), hombres y mujeres de todas las disciplinas científicas.

En el trabajo de campo se entrevistaron a 84 investigadores en cuatro estados: D.F., Puebla, Hidalgo y Tlaxcala a partir de cuotas muestrales obtenidas del propio listado del SNI para cada entidad federativa, ponderando (según la importancia de las instituciones de investigación existentes por entidad) en función del número de SNI's que tienen contratados. Las instituciones atendidas fueron12: UNAM, UAM, CINVESTAV y Politécnico Nacional en el DF; la BUAP, UDLA, IBERO, INAOE y Colegio de Posgraduados en Puebla; la UAT y el Colegio de Tlaxcala en el estado de Tlaxcala y la UAEH en Hidalgo 


\section{LA VISIÓN MITOLOGIZADA DEL INVESTIGADOR Y DE SU MERCADO DE TRABAJO}

Poco se conoce sobre las peculiaridades del trabajo de los investigadores y una de las principales causas que alimentan este desconocimiento es el no reconocer que ésta es, en primera instancia, una actividad laboral. A cambio, la sociedad se ha conformado con una representación mítica acerca de lo que es un investigador. Esta representación sucedánea está llena de atributos ideales que por supuesto rebasan los atributos que normalmente tienen el grueso de los investigadores comunes, de los investigadores de "carne y hueso".

Existen al menos dos visiones idílicas claramente diferenciables: una, conforma una imagen del investigador a partir de una condición de excepcionalidad genética; en ese sentido, un investigador es, por definición, "un genio" y por ende, diferente al resto de los mortales. La otra visión también ubica al investigador como un ser diferente al resto de los mortales pero amparada no en motivos genéticos sino en una determinación social, que ha logrado su excepcionalidad por acción de su capital social, en muchas ocasiones ligado a un origen de alcurnia, un ser proveniente de familias con amplia tradición intelectual y con recursos económicos suficientes para dedicarse por años al estudio. Estas dos visiones sirven de mampara que oculta no solo la dimensión humana ordinaria de los investigadores sino además los avatares de sus relaciones laborales.

Algunos investigadores alimentaron -con sus acciones y con su figura- esta visión excéntrica, pero ninguno, a lo largo de la historia, al menos desde Galileo hasta la actualidad, ha dejado de llevar trayectorias biográficas similares a las de cualquier ser humano. Es cierto que con el desarrollo de las ciencias modernas hubo un predominio de científicos que provenían de familias acomodadas económicamente y con amplia tradición intelectual, pero estos rasgos dejaron de tener la misma importancia en los tiempos actuales. Sin negar la genialidad que pueden llegar a desarrollar los científicos, es indudable que al final de cuentas, la laboriosidad desplegada en los ambientes laborales de los centros de investigación van definiendo las diferencias entre ellos. Hay una fuerte asociación entre la obra científica de calidad y la laboriosidad. Los investigadores son, sin duda uno de los segmentos laborales más laboriosos.

Hay que advertir que los atributos percibidos como individuales, contradictoriamente tienen un origen social, estas capacidades son producto de una historia social y aunque algunas puedan estar adicionadas de componentes genéticos muy personales que potencian su brillantes, la gran mayoría de las trayectorias exitosas resultan de procesos históricos y sus resultados no de- 
penden sólo del esfuerzo y tenacidad individual sino también de condiciones y procesos colectivos en los que han estado involucrados los investigadores.

A partir de la consideración de que los científicos son seres absolutamente análogos al resto de los humanos, también estamos en posibilidad de reconocer que la actividad científica puede ser considerada como una especialidad profesional equivalente a cualquier otra en el espectro de actividades laborales, se puede estudiar de manera similar a como se estudian otros tipos de mercado.

La investigación en ciencias sociales poco a poco ha incluido dentro del espectro de sus temáticas el de las relaciones laborales de los científicos o investigadores y han involucrado también el análisis de los sujetos que la realizan, su comportamiento y percepciones; en el marco de una actividad con al menos dos sentidos, uno, el de su realización intelectual muy ligada a lo simbólico y, el otro, como una actividad de reproducción económica, eminentemente material.

Lo que se llama "comunidad científica realmente existente" es la contraparte de esa imagen ideal, mítica que marca un juego de caminos entrelazados donde se superponen tanto las visiones plenas de simbolismo con las necesidades materiales de reproducción. Este doble sentido de la acción laboral no es exclusiva de los investigadores, de hecho cada sujeto ligado al mercado de trabajo despliega simultáneamente a las funciones de reproducción argumentos de trascendencia que dan sentido a su actividad laboral, sin embargo, con los investigadores están más a la mano imágenes y arquetipos impregnados de una mayor idealidad.

Los estudios de Larissa Lomnitz han ayudado a la desmitificación de la actividad de los investigadores en México, ella y sus discípulos han estudiado con detenimiento algunos de los centros de investigación de la UNAM desde la década de los setenta y, desde su visión antropológica ha percibido lo que ella identifica como proceso de construcción de la "identidad" del científico mexicano. Desde su punto de vista, no basta con el dominio de una serie de conocimientos especializados, es necesario también que se llegue a internalizar un conjunto de creencias y valores prescriptivos fundados en una interpretación compartida a nivel social acerca de lo que es hacer ciencia, de lo que es ser un científico y de cómo se llega a ser un científico. Este conjunto de normas y creencias formales e informales es lo que ella misma identifica como "ideología científica". Desde su perspectiva y nosotros la compartimos, México estaría en la fase de construcción de una ideología científica propia que estaría vinculándose al quehacer individual y colectivo de los investigadores.

Algunos puntos que esta autora ha encontrado como factores ideológicos compartidos entre los investigadores con los que ella ha trabajado a lo largo 
de 20 años están vinculados a concepciones generales acerca de la ciencia y el universo y son (LOMNITZ; 2008):

a) El hombre puede conocer y controlar la naturaleza

b) La ciencia y el método científico son los instrumentos que permiten al hombre comprender la naturaleza

c) La ciencia es el único instrumento de conocimiento para la comprensión de la naturaleza

d) La meta más importante del científico es la búsqueda de la verdad y de esto obtiene su más alta gratificación

e) El conocimiento de la naturaleza es normalmente bueno y puede salvar a la humanidad

f) El conocimiento y la búsqueda de la verdad trascienden al individuo y constituyen el logro de la comunidad entera.

Esta definición es interesante pues indica la fortaleza que aún tiene la idea de certeza como elemento de validación científica y el sentido -casi ontológico- de la ciencia como un elemento externo, transformador y positivo; pero más allá de que entre los científicos mexicanos se compartan conceptos de ciencia más bien tradicionalistas, es evidente que comienza a socializarse un corpus de identificación con lo que ellos identifican por "realmente científico". Una de las polémicas más enconadas en este momento tiene que ver con las diferencias conceptuales entre los que sostienen ideas de la ciencia como las halladas y descritas por Lomnitz y otro grupo de científicos generalmente más vinculados a las ciencias sociales que plantean visiones más abiertas y modernas. Esto da evidencia de la existencia de una sociedad científica activa, en proceso de consolidación.

La constitución de una sociedad de científicos contemporáneos en México, provista de atributos cosmopolitas, está dejando atrás las visiones míticas y se encamina al levantamiento del "primer piso" de científicos en posibilidad de poseer una ideología propia, nacionalista, con una visión de la ciencia más abierta y moderna y esto tiene que ver especialmente con su condición laboral. 


\section{EL MERCADO DE TRABAJO DE LOS INVESTIGADORES Y LAS DESIGUALDADES}

La constitución del mercado de trabajo de los investigadores no se debe ver mecánicamente como la simple generación de plazas dentro de los centros de investigación, paralelamente se definen propósitos y resultados que le dan especificidad laboral dentro de un marco institucional de alto control. En los trabajos de Lomnitz se plantea que los centros de investigación, y en general el sistema de evaluación de los resultados de investigación se ha sometido a un conjunto de reglas muy estrictas al amparo de la búsqueda del rigor.

[...] Se espera de todos los científicos algún grado de originalidad y creatividad, pero estas cualidades deben estar apoyadas en una estructura controladora, fuerte y consistente. La adquisición de hábitos y habilidades de trabajo es una de las formas más simples del control conductual y estarían dirigidas a neutralizar tendencias hacia el desorden, ineficiencias y holgazanería [...] (LOMNITZ; FORTES, 1981).

Las formas y estructuras normativas de la actividad de investigación son un proceso lento de socialización que a veces no alcanza plena definición cuando el segmento de mercado involucrado no es muy antiguo. Lo que se podría considerar el inicio, o el momento de surgimiento, del mercado de trabajo de los investigadores en México ocurre en la capital de la República no hace más de cuatro décadas y al amparo del crecimiento -casi explosivo- de los espacios universitarios. Algunos estudiosos del tema plantean el inicio de la institucionalización de este segmento de mercado justo en el momento de creación del Consejo Nacional de Ciencia y Tecnología en 1973 (SÁNCHEZ DAZA, 2000) y simultáneamente a su consolidación y limitada descentralización se construye una estructura controladora que permite la formación de los recursos humanos especializados en "las artes" de la investigación.

Se instituye no sólo el mercado como espacio de oferta y demanda, también se forman generaciones nuevas de investigadores con elementos de identidad que permiten hablar de una profesionalización de la investigación. Es esta misma identidad la que impone un marco de lógicas de pensamiento y de concepciones acerca de esta actividad y se materializa en una concepción compartida que facilita el rigor en términos teóricos y técnicos. La popularización de la investigación no se tradujo en la pérdida de exigencia y de rigor. 
En el imaginario colectivo compartido entre los investigadores mexicanos es claro que se espera del científico que sea trabajador y extremadamente entregado. Que viva en su trabajo y para él.

Se trata de un segmento de mercado de reciente creación, altamente centralizado a nivel territorial pues el $45 \%$ de los investigadores reconocidos en el SNI labora en el DF. Pero también hay una centralización en término de instituciones educativas pues en la UNAM trabaja el 23\%; en el IPN el $9 \%$ y en la UAM otro $6 \%$ con lo cual tenemos que tan sólo con estas tres instituciones ya se concentra el 38\% de todos los investigadores reconocidos en México. La investigación en México se realiza fundamentalmente dentro de las universidades públicas y privadas.

Uno de los espacios donde se hace investigación de manera preferencial es en los posgrados, aqui es donde se puede identificar con mayor facilidad a los centros de investigación, aunque existen otros espacios donde también se construye conocimiento. La extraordinaria dinámica de crecimiento de este mercado se puede entender a partir de su asociación con el crecimiento explosivo del nivel de posgrado en nuestro país a partir de la década de 1990, nivel que por cierto ha sostenido este ritmo de crecimiento hasta la fecha, según se aprecia en el Cuadro 1.

\begin{tabular}{|l|c|c|c|}
\hline Ciclo escolar & ESPECIALIDAD & MAESTRÍA & DOCTORADO \\
\hline $2004-2005$ & 31,314 & 106,457 & 13,081 \\
\hline $2005-2006$ & 31,727 & 108,722 & 13,458 \\
\hline $2006-2007$ & 34,898 & 111,970 & 15,135 \\
\hline
\end{tabular}

Cuadro 1: MATRICULA DE ALUMNOS DEL POSGRADO EN MÉXICO

Fuente: ANUIES (2007). Anuario estadístico. México

Sin embargo, el crecimiento del posgrado no ha ido aparejado de las mejores condiciones de trabajo para todos, especialmente de los que se están incorporando en el último lustro. Las plazas de docencia ocupadas en la impartición de posgrados, idealmente debían ser recursos que en parte cubrieran su labor total investigando y lo deseable sería que tuvieran el reconocimiento del SNI. Desafortunadamente, el subsistema de posgrado no dispone de plazas donde se pudiera exigir el cumplimiento a cabalidad de la investigación ya que también contrata personal docente por medio tiempo y tiempo por horas. 
Desafortunadamente, el tipo de contratación en este subnivel de posgrado aún presenta pocos alicientes para su expansión, ya que la relación de docentes de tiempo completo respecto del total se mantiene históricamente por abajo del $50 \%$ en general. Aunque es obvio que algunas instituciones cuentan con posgrados muy consolidados y tienen la totalidad de su planta contratada por tiempo completo e investigando.

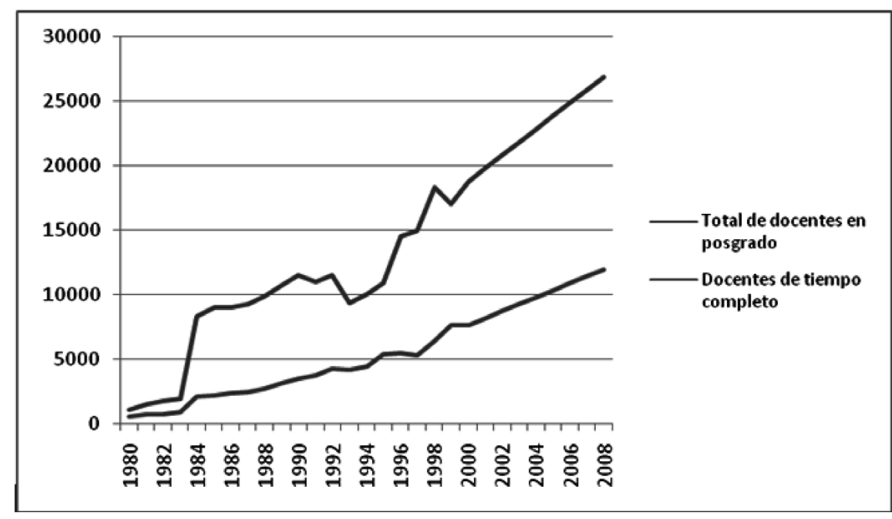

Gráfico 1: Docentes contratados en Posgrado

Fuente: ANUIES. (1999). Personal ocupado. México

Este "boom" de los posgrados ha facilitado la inserción de las mujeres en este mercado. La participación de las mujeres ha crecido de manera importante aún cuando su presencia actual sea del $32.5 \%$ de las plazas de investigador con reconocimiento SNI. Este dato sube ligeramente al $36.8 \%$ en la base de investigadores con beca del PROMEP. En la muestra de investigadores que se hizo para los estados bajo estudio el valor de participación femenina se ubica en e1 $31 \%$. Es decir, hay una tendencia muy similar en las tres fuentes. Un tercio del mercado de trabajo de los investigadores es ocupado por mujeres.

Según CONACYT para 2008, existen 328 centros de investigación -tanto públicos como privados- en México. El $73.5 \%$ de estos centros son de origen público; el 25.6\% son privados (CONACYT, 2008). Estos centros son de muy diversos tamaños lo cual se traduce en diferentes capacidades de trabajo; se encuentran desde los centros "macro" de las universidades hasta centros "micro" con dos o tres miembros reconocidos (que cubren al 30 por ciento del total, que es una proporción muy alta). La importancia del tamaño de los centros en los niveles de eficiencia de la investigación no se tocara en este artículo, lo que interesa destacar es la existencia de posibles espacios de crecimiento del 
mercado ya que esa heterogeneidad funcional de los centros de investigación lleva a hipotetizar acerca de la posibilidad de mantener el crecimiento de este segmento de mercado.

El crecimiento del número de investigadores en México es muy importante a partir de la década de los noventa, su crecimiento es tan vigoroso que su número llega a duplicarse en tan solo un lustro (Ver Grafico 2)

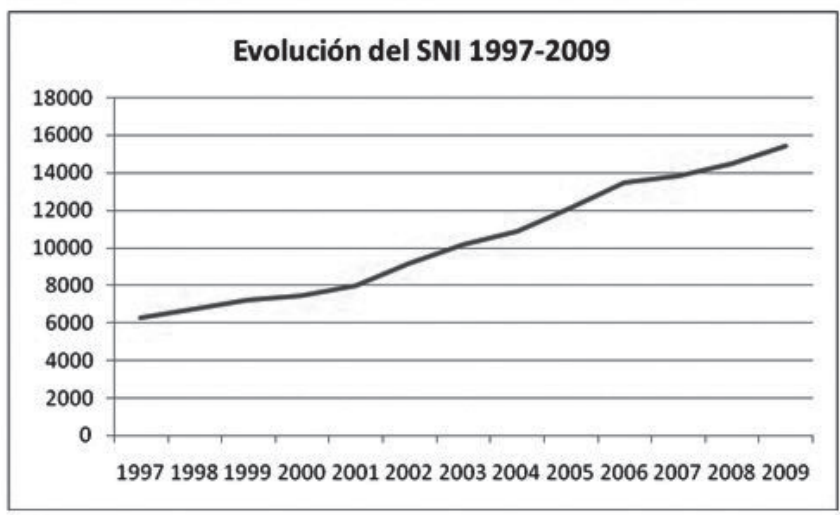

Grafico 2: México. Crecimiento del número de investigadores en el SNI Fuente. CONACYT (2009). SNI. México

El mercado de los investigadores se manifiesta como un espacio de alta competencia pero perfectamente diferenciada y sujeto a un conjunto de normas escritas que incluye a otro "paquete" de normas y acuerdos no escritos, implícitos, fijados por los investigadores con mayor antigüedad.

Existe competencia pero está limitada a la misma especialidad, de la misma manera que existen grupos no competitivos en otros segmentos del mercado; es decir, de la misma manera que los pintores no compiten con los albañiles, ni los carniceros con los abogados, un físico del estado sólido no compite con un químico orgánico o un antropólogo. Aún entre los investigadores existen grupos no competitivos, las especialidades levantan barreras para el movimiento dentro del mercado.

Las barreras del mercado de investigadores van limitando la competencia pero a cambio se generan secciones de mercado similares a las que Piore denomina los "mercados internos" (PIORE; DOERINGER, 1985), con una alta restricción al ingreso de "elementos externos" o "no estandarizados" (en el sentido de no poseer las mismas credenciales, de no compartir los mismos enfoques). 
Otra forma de entender las restricciones al ingreso en ciertos espacios del mercado de investigadores se podría hacer a través de lo que Weber denomina "cierre social" y que plantea una alta capacidad de elección en manos de los investigadores más antiguos. Los puestos vacantes se ocupan en muchas ocasiones según la antigüedad que ya tienen otros investigadores del mismo equipo, o con los aprendices que están más ligados a las antiguas generaciones o a la institución. Dados los mecanismos de ingreso que se han podido identificar, podría decirse que estamos ante un mercado altamente institucionalizado.

Valdría la pena recordar también la visión de Clark Kerr en cuanto a las normas que operan en cualquier mercado y que se podrían recuperar para entender la dinámica impuesta por una normatividad explicita e implícita que existe en el mercado de los investigadores. Kerr sostiene que en los mercados no existen "leyes naturales", se trata finalmente de las decisiones que han tomado previamente los sujetos pero que se han sedimentado en forma de normas o leyes en las cuales no se identifica la influencia de los investigadores de generaciones anteriores...."... "El imperio de la ley no es sino el imperio de los hombres, aunque indirectamente [...]." (KERR, 1954).

El mercado de los investigadores esta institucionalizado, las instituciones definen fronteras entre las diferentes áreas del mercado y las hacen más específicas y difíciles de cruzar. Definen los puntos de competencia y los personajes y grupos susceptibles de competir

Como cualquier mercado institucional, a su interior se reproducen esquemas de desigualdad que en muchos casos se manifiestan de manera muy sutil pero efectiva ${ }^{2}$. En este mercado son ubicables rasgos de desigualdad asociados fundamentalmente al género y a la edad. En los datos del SNI y de la encuesta levantada en cuatro estados se encontró que en términos de género, las mujeres encuentran obstáculos que sólo permiten que el 32.5\% (CONACYT, 2008) de ellas obtengan un empleo y sean reconocidas por este sistema. En cuanto a los jóvenes investigadores, menores de 40 años, tan solo el 20.6\% logra una ubicación en este sistema (UAM-BUAP, 2009).

1 Weber acuñó el concepto de "cierre social" para designar los mecanismos y procesos mediante los cuales un grupo mantiene el acceso privilegiado a un recurso y excluye a los que no pertenecen a ese grupo. Los dos mecanismos básicos de la desigualdad son: la apropiación del valor excedente producido por el trabajo ajeno y la obtención de un beneficio mediante el control del acceso a un recurso o ventaja (acaparamiento de oportunidades -exclusión-) (ARTILES; KOHLER 2007).

2 La desigualdad no es un derivado de alguna esencia humana inmutable, una cuestión natural o un imperativo estructura, ni siquiera el producto de las diferencias en las habilidades de los individuos sino una construcción histórica social. En cada sociedad y en cada época, existen diferentes tipos de desigualdades, también se modifican los diferentes grados de desigualdad e igualdad lo mismo que los factores que la generan por lo que hay que especificar las especificidades de cada caso (REYGADAS; 2008). 
En sociedades complejas es de esperar que existan muchas diferencias y disparidades, sin embargo lo interesante es percibir como se le da a esas diferencias una especie de "carta de naturalidad" en el sentido de que se le llega a atribuir tal legitimidad que se justifican esas mismas diferencias.

En nuestra encuesta sólo el 2\% reconoció explícitamente la existencia de desigualdades, aunque encubiertas en el ambiente "neutro" de la normatividad universitaria. Fueron las investigadoras con mayor edad y experiencia las que indicaron esta situación. Otro $2 \%$ reconoció la posibilidad de que ciertos ambientes universitarios -competitivos y hostiles- puedan inhibir, en términos personales, a algunos investigadores jóvenes y a investigadoras. E1 96\% que obviamente incluye tanto a hombres como mujeres, en casi la misma proporción, no reconocieron la existencia de obstáculos por género o edad, es más, plantearon la existencia de condiciones neutras y equitativas en los ambientes de la investigación,......lo que hay que vencer es la enorme competencia existente y esto, lo hace sólo el que es competente, al margen de si es mujer u hombre (UAM-BUAP, 2009).

Ante un ambiente de mercado que justifica la existencia de diferencias, resulta natural que no aparezcan explícitamente las disputas que entablan los actores que están involucrados en relación a la equidad o inequidad. Hasta el momento no se tiene registros de investigadoras o de investigadores jóvenes que demanden colectivamente mayores oportunidades. Al parecer, las amarguras del desplazamiento o exclusión original son compensadas u olvidadas en cuanto un investigador es aceptado en algún centro.

Los procesos simbólicos son un componente fundamental de la construcción de la igualdad y la desigualdad en los mercados de trabajo. La distribución de los bienes y servicios nunca sigue una lógica racional culturalmente neutra ni se ajusta al funcionamiento de un mercado perfecto, sino que pasa por los filtros de la cultura, de las costumbres, cuyos procesos de valorización, diferenciación y jerarquización inciden en la determinación de la cantidad y la calidad de los beneficios que cada individuo y cada grupo recibe en la sociedad,

[...] la desigualdad no es algo natural sino redes de privilegios y perjuicios que se establecen o autorizan por el consentimiento de los hombres. Los mercados perfectos no existen, siempre están incrustados en contextos sociales e institucionales mediados por relaciones de poder y procesos culturales específicos que pueden incrementar o reducir la desigualdad (REYGADAS, 2008).

3 El estudio de la desigualdad tiene como punto de partida el principio de que desde un punto de vista ontológico. Todos los seres humanos somos iguales, todos tenemos los mismos derechos fundamentales, ninguna vida vale más que otra y nadie es superior o inferior a los demás - pero esta igualdad básica está en tensión con una profunda desigualdad existencial 
El mercado de los investigadores es un mercado pleno de desigualdades. Hay una enorme disparidad de ingresos, existen barreras de acceso y barreras de ascenso, existen criterios de valoración no definidos por la productividad, etc. lo extraño es que esta situación ha alcanzado el estatus de "normal" y ya no es apreciable por todos los investigadores, pareciera que se ha llegado a un consenso en torno a una determinada distribución, que siempre será contingente y cambiará cuando se modifiquen las condiciones y posiciones, pero en esencia seguiría todo igual. Hay algunas desigualdades que la mayoría de las personas consideran "justas" o bien que se ejercen en un cierto nivel que parece razonable.

En este momento, por poner un ejemplo, ya no llaman la atención las condiciones de exclusión vertical de las mujeres dentro del sistema nacional de investigadores. A nadie extraña esa anormal distribución de reconocimientos que se hace, porque está encubierta y justificada por un esquema de evaluación en apariencia "igualitario".

Ya se había señalado antes la condición de desigualdad permanente en el ingreso de las investigadoras a los centros de trabajo que son básicamente universidades públicas y privadas. Se comentó ya que las mujeres han llegado a ocupar tan sólo el $32.5 \%$ de las plazas existentes en este mercado, pero es una situación que se ha mantenido así desde hace dos décadas y se conserva en esos términos a pesar de la creciente feminización de la investigación en todas las disciplinas, esto es lo que con el tiempo se convierte en una distribución "normal", "aceptable". En el siguiente gráfico se presenta la brecha existente entre investigadores e investigadoras reconocidas en el SNI.

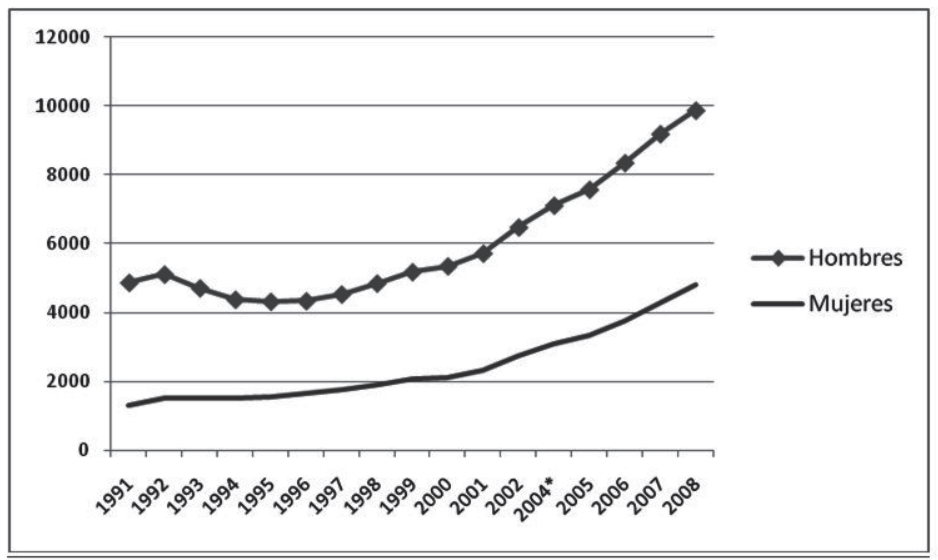

Gráfico 3: Número de investigadores e investigadoras en el SIN Fuente: CONACYT. (2008). Informe anual. México 
A las evidentes restricciones que existen para el ingreso de las mujeres dedicadas a la ciencia en su mercado natural de trabajo, existe un segundo tipo de exclusión: la exclusión vertical. La OIT deja claro que a una gran mayoría de las mujeres se les ha dificultado alcanzar puestos de alta jerarquía, esta es una de las formas de exclusión que algunos estudiosos del tema denominan "techo de cristal" (WIRTH, 2001).

El techo de cristal de las investigadoras, se manifiesta como una imposibilidad de participar igualitariamente en todos los niveles jerárquicos de reconocimiento en el SNI. En este sistema hay una clasificación de cuatro niveles jerárquicos: los candidatos a investigador son los más jóvenes (menores de 40 años) y con menor experiencia y producción; el nivel I, donde se agrupa la mayor cantidad de investigadores y los niveles II y III, con los investigadores más consolidados, de mayor reconocimiento académico, obviamente con una participación más restringida y que obtienen mayores apoyos. Las aportaciones económicas que en forma de becas se otorgan a los investigadores reconocidos dentro de este sistema varían según el nivel que ocupan, siendo las más altas las de los mayores niveles.

\begin{tabular}{|l|c|}
\hline Nivel & Investigadoras \\
\hline Candidato & 20,3 \\
\hline Nivel I & 58,1 \\
\hline Nivel II & 17,3 \\
\hline Nivel III & 4,4 \\
\hline
\end{tabular}

Cuadro n. 2 Participación de las investigadoras en el SNI, egún nivel de reconocimiento (\%)

Fuente: CONACYT (2008). México

El porcentaje acumulativo de las investigadoras en los niveles de candidato y nivel I suma el $78.4 \%$, lo cual indica que prácticamente las tres cuartas partes de las investigadoras están en la escala más baja de reconocimiento. Para el resto, la pelea por el reconocimiento se hace aún más complicada.

Es factible para las investigadoras en México ascender en la escala de reconocimiento en los dos primeros niveles, es decir, en los de candidato a 
investigador y en el nivel I; incluso, son más las mujeres que se ubican en el nivel I que los hombres. Sin embargo, la situación se complica para ellas cuando se avanza al nivel II y no se diga cuando se trata de ascender al más alto; el codiciado nivel III en donde solo es factible que lleguen 4 mujeres de cada 100 investigadores.

Es preciso reconocer los esfuerzos individuales o grupales para salir adelante, para vencer los obstáculos, para "no quedarse atrás" o "no quedarse afuera". Esto es lo que se conoce como "resiliencia", es decir, la capacidad para afrontar una situación desventajosa ${ }^{4}$.

El énfasis en la resiliencia y en la resistencia permite des-victimizar a quienes experimentan la exclusión y la desigualdad, ayuda a verlos como agentes con mayor o menor capacidad para afrontarlas y no como sujetos disminuidos que requieren ayuda y asistencia social. Esta noción de resiliencia es especialmente útil para entender las estrategias de las investigadoras para ingresar y permanecer dentro de su mercado de trabajo.

Para ser justos, es preciso reconocer que se percibe en todos los investigadores mexicanos -hombres y mujeres- una muy alta capacidad de resiliencia pues sobran los ejemplos -en ambos sexos- donde los investigadores han logrado superar las condiciones más adversas para llegar a una situación diferente, sobre todo por lo que implica provenir de hogares humildes. En el mercado de los investigadores son pocos los que han llevado una trayectoria laboral tranquila y fácil, pero a las mujeres -con sus obvias excepciones- les cuesta más trabajo; es por ello que en este apartado nos orientamos de manera preferente a las cualidades desplegadas por las investigadoras

En las entrevistas sostenidas, especialmente con investigadoras, es posible percibir la resiliencia en forma de estrategias de lucha y ascenso que las ha llevado incluso, a ser más exitosas que sus pares varones. Las investigadoras consolidadas y con una cierta antigüedad ocupan mayoritariamente mejores posiciones laborales y profesionales (jefas de departamento o área, directoras de centros de investigación, coordinadoras de proyectos de amplio alcance, etc.) que sus colegas hombres. Esta situación incluye a sus parejas que generalmente ocupan posiciones de menor responsabilidad e ingreso.

El posicionamiento de las mujeres dentro del ámbito de la investigación, aún enfrentando restricciones ha modificado el panorama en términos de la

4 La resiliencia ha sido analizada como un recurso psicológico de los individuos para reponerse de un trauma de una infancia infeliz. Pero también puede ser analizada desde el punto de vista sociológico y antropológico como la capacidad creadora y restauradora de los agentes individuales y colectivos para afrontar la adversidad, la pobreza y la exclusión...(REYGADAS, 2008). 
construcción de nuevos tipo de relaciones y en la ampliación de temáticas y enfoques. La resiliencia se manifiesta también a través de las rupturas que de manera individual van adoptando las investigadoras. Las investigadoras que entrevistamos han realizado rupturas respecto a los patrones imperantes, una muy importante es la que toca al patrón de reproducción o fecundidad pues el promedio de nacimientos entre ellas es de 1.5 en toda su vida, patrón que es totalmente diferente al de sus madres que tuvo un promedio de 4 nacimientos en promedio.

A esto hay que adicionar la ruptura de los patrones de relación al interior de la familia con sus parejas y sus hijos. La resiliencia como estrategia de defensa puede tener expectativas de largo plazo, como ocurre con aquellas investigadoras que prefieren iniciar (aunque en algunos casos nunca hubo espacio para la decisión) de manera relativamente tardía su carrera científica; dando la posibilidad de que sus hijos crezcan y terminen sus estudios y ya luego ellas estén en posibilidades de reiniciar estudios truncos o regresar a trabajar, o dedicarse con mayor entrega a la investigación y en esos casos su actividad pronto se traduce en resultados importantes. Otra forma de resiliencia implica el no esperar y de inmediato plantear "ajustes" que se traducen en diversos tipos de transacciones que deben hacer con sus parejas, con sus colegas y con ellas mismas para compaginar sus intereses profesionales con los intereses más personales que provienen de la familia y de su propia cultura, en ello juegan las tradiciones e imaginarios sociales compartidos acerca de su forma de realización como madres, como esposas y como nueva familia.

El mercado de trabajo de los investigadores muestra una dinámica de cambio que supera a cualquier otro mercado laboral, no solo en cuanto a su crecimiento sino en sus condiciones de ingreso y en su gradual pero constante inclusión de nuevos buscadores. A pesar de ello, la desigualdad persiste pero ocurre en un ambiente aparentemente igualitario, donde formalmente todos, absolutamente todos, están en posibilidades de aspirar y lograr cualquier plaza, cualquier puesto, cualquier reconocimiento; sin embargo esto realmente no es así.

\section{DESIGUALDAD Y ORIGEN SOCIAL DE LOS INVESTIGADORES EN MÉXICO}

La consolidación de la actividad científica como una relación laboral, ha provocado una profunda transformación en cuanto al origen social de los investigadores. La "popularización" de la actividad de investigación ha permitido 
la irrupción de investigadores que provienen de clases con bajos recursos económicos; sin tradición cultural familiar, con estudios en escuelas públicas. Ha habido una ampliación de las oportunidades para investigadores con un origen social y cultural más popular, de menores recursos económicos.

Algunas investigaciones realizadas -a mediados de la década de los setenta y ochenta- en instituciones del centro del país, indagaron sobre la formación de los científicos y daban cuenta de otra situación (LOMNITZ; FORTES, 1981). En ese momento, testimoniaron que los estudiantes de aquellas décadas que se interesaban por iniciar carreras profesionales en la ciencia seguían una "tradición familiar" y pertenecían a una clase social media o "acomodada". Provenían, además, de escuelas particulares y sus padres solían ser profesionistas (IZQUIERDO, 2006).

Para el año 2006 ya es apreciable un cambio fundamental en cuanto al perfil biográfico y laboral de los investigadores en México. La propia Isabel Izquierdo en su análisis de los investigadores de Ingeniería y Física de la UAEM ya detecta cambios en el sentido de que:

[...] Nuestro estudio por su parte (se llevó a cabo en una institución estatal en 2004), con una temporalidad y escenario distintos al de la autora [se refiere a Lomnitz], reveló que los investigadores, en su mayoría, provienen de familias con bajos recursos económicos y de padres no profesionistas [...] (IZQUIERDO, 2006).

El hallazgo de Izquierdo coincide con lo que se encontró en este estudio, aunque ahora se puede hacer extensivo a 12 IES de cuatro estados de la república y para investigadores de todas las disciplinas científicas. Respecto al origen familiar, se encontró que el $78 \%$ de los investigadores entrevistados proviene de hogares donde los padres no tienen estudios profesionales. Además, han realizado sus estudios de maestría $(77 \%)$ y doctorado $(60 \%)$ en escuelas públicas, básicamente en el D.F.

Del doctorado, un $40 \%$ lo hizo en el extranjero y además, el $90 \%$ de los investigadores entrevistados han logrado ese doctorado como caso único de su familia (son los únicos doctores en su familia pero también coincide con ser los primeros doctores de su colonia, o de su pueblo). Es por ello que planteamos que esta generación de investigadores - por su origen y por su edad- constituye el "primer piso" de la investigación en México.

En cuanto a la formación de los investigadores, tenemos que poco más del $75 \%$ tiene el doctorado y casi un $20 \%$ han hecho el posdoctorado. Es muy bajo el número de investigadores con nivel de maestría (5\%) y aún menor de 
aquellos que sólo tienen la licenciatura. Es evidente el efecto de "atracción" hacia grados superiores de los programas SNI y PROMEP.

La edad de los investigadores ya resulta un punto preocupante, pues en promedio los investigadores mexicanos andan en sus 48 años. Aún cuando hay una dotación de investigadores más jóvenes no deja de ser alarmante el futuro -no muy lejano- de este mercado pues es una edad biológicamente avanzada y sin mecanismos institucionales de recambio generacional.

Si hablamos de la experiencia laboral de los investigadores entrevistados, encontramos que coincide con la edad promedio. La experiencia se ubica mayoritariamente entre 10 y 20 años (42\%), pero hay una porción importante a ambos lados de este valor. El 27\% de investigadores tiene menos de 10 años de experiencia y el $25 \%$ tienen entre 20 y 30 años de experiencia

Se había comentado al inicio de este artículo que los investigadores son un segmento que se caracteriza por su laboriosidad. En general podemos destacar que la cantidad de horas diarias de trabajo promedio de los investigadores entrevistados es de 10.2 horas, lo cual habla de una alta capacidad de compromiso con su actividad o de una muy eficiente capacidad de control de las estructuras institucionales pues generalmente esta decisión de mayor trabajo al formalmente instituido aparece como una disposición voluntaria, personal, que no está sujeta a más presión que la de sus propios proyectos y entrega a su labor. Esta punto de vista quizá pueda modificarse en cuanto se aprecie en su conjunto con la composición de la estructura salarial.

Respecto a su pareja, el $74 \%$ casa con profesionistas y tienen en promedio menos de 2 hijos, lo cual habla de una transformación en los patrones de reproducción pues casi todos ellos provienen de familias con un promedio de cuatro hijos.

La conformación del mercado de trabajo de los investigadores hacia la década de 1970 y su impresionante crecimiento en las siguientes dos décadas, lo habilitó de rasgos netamente laborales en los que pudieron insertarse exitosamente nuevos cuadros de origen social diferente. Los cambios en las orientaciones educativas y la búsqueda de rendimientos con mayor calidad posibilitaron el advenimiento de investigadores profesionales que ya no se correspondían con el perfil de las décadas anteriores.

En el proceso de consolidación de este mercado hubo una impresionante apertura al ingreso, en ese momento de jóvenes dedicados a la ciencia -tanto hombres como mujeres-, aunque muy pronto acuso procesos de rigidización provenientes del juego de relaciones a nivel social y que reprodujeron los esquemas de desigualdad persistentes a nivel social. 


\section{PERFIL DEL MERCADO DE TRABAJO DE LOS INVESTIGADORES}

En este apartado se hará un perfil muy general y descriptivo del mercado de trabajo de los investigadores. Disponemos de información para cuatro estados pero se obtiene una impresión que no es muy de la que se percibe en el resto de los estados donde se realiza mayoritariamente investigación universitaria.

Dada la importancia poblacional de México y dada su importancia económica, el tamaño de su mercado de investigadores es aún sumamente reducido ${ }^{5}$, lo cual nos habla de la necesidad imperiosa de su crecimiento, pero además de su consolidación. Si estamos de acuerdo con Sánchez Daza (2000) de que la década de los setenta fue el momento de institucionalización de la actividad científica en nuestro país, estaríamos planteando ahora la necesidad de una segunda etapa de institucionalización que se podría caracterizar por la búsqueda de un crecimiento más armónico y racional de la investigación en lo referente a los espacios geográficos y en cuanto a las instituciones, además del logro de una estructura salarial de mayor seguridad, una organización institucional con criterios de apoyo más igualitario y con normas claras y, sobre todo, con una política científica nacional de largo plazo que busque la constitución de una ciencia realmente mexicana.

\section{OBSTÁCULOS FUNDAMENTALES EN EL MERCADO DE TRABAJO DE LOS INVESTIGADORES}

El principal obstáculo del mercado de investigadores es su extremada centralización, pues ha generado deformaciones que impiden, por un lado un mayor crecimiento, por otro lado un crecimiento más racional. Derivado de la misma centralización se percibe un bloqueo al desarrollo de las zonas con menor actividad científica. Finalmente la centralización también ha deformado los esquemas salariales. Veamos con detenimiento cada uno de estos puntos.

\subsection{La centralización como obstáculo al crecimiento del mercado de investigadores}

Se menciono con anterioridad que en el DF se encuentran laborando casi la mitad del total de investigadores reconocidos de México y en este mismo

5 Compárese con el dato que indica que para finales de la II Guerra Mundial, en los EUA se tenía un registro de 50 mil investigadores, mientras las estimaciones en México son de 35 mil investigadores (CAMPOS, 2009). 
territorio se encuentran las tres instituciones educativas con mayor capacidad de contratación de investigadores de todo el país.

Esta extraordinaria centralización se ha mantenido bajo el argumento de que permite la consolidación eficiente de recursos, materiales y humanos; sin embargo ha llegado a un punto que podría considerarse de saturación espacial, en el cual cada vez será más difícil obtener recursos para contratar a un investigador y resolver la parafernalia que lo acompaña, pero especialmente el vinculado a su espacio de acción y a la infraestructura que implica.

Cada institución de manera individual enfrenta anualmente mayores dificultades con la aprobación de mayores recursos, incluso en el supuesto de que el gobierno mexicano hiciera mayores inversiones en $\mathrm{I}+\mathrm{D}$, ya es difícil proponerse que crezcan más y especialmente en el mismo territorio.

La racionalidad en el crecimiento también se ve obstaculizada por la concentración de recursos en la capital de la república. De esa manera es que tenemos a 9 estados donde la actividad de investigación es tan raquítica que no llegan a tener ni siquiera cien investigadores con reconocimiento. Esta condición de debilidad de algunos estados no es culpa exclusiva de la concentración sino que influye un cúmulo de razones y omisiones responsabilidad de los gobiernos locales. Sin embargo, esta misma debilidad les impide negociar o cabildear de manera más eficiente en los momentos de definición de los presupuestos en el Congreso. Esta dificultad se agranda con la condición de creciente fortaleza de las regiones e instituciones con mayor concentración.

\subsection{La centralización como obstáculo al crecimiento de los más débiles}

Los estados con menor desarrollo de la investigación adolecen de instituciones donde se realice investigación pero además carecen de los recursos humanos de calidad y en el número mínimo para emprender proyectos de desarrollo con posibilidades de cambiar el rumbo de esta actividad.

Mientras el mercado de trabajo de la investigación se concentra básicamente en 10 estados (casi el 80\%), tenemos una cantidad mayor de estados donde su actividad de investigación no representa ni el 3\% de la actividad nacional medida como numero de investigadores reconocidos

Podemos ubicar 12 estados de la República mexicana en los cuales se puede promover una ampliación de su mercado de investigación: 1) Morelos, 2) San Luis Potosí, 3) Durango, 4) Zacatecas, 5) Guerrero, 6) Campeche, 7) Oaxaca, 8) Tlaxcala, 9) Baja California Sur, 10) Quintana Roo, 11) Nayarit y 12) Colima. 


\subsection{La concentración como deformadora de la relación salarial}

La estructura de los salarios de los investigadores está totalmente fraccionada en múltiples segmentos, de modo que podemos hablar de un salario base que cada vez tiene menor peso en el ingreso total y un ingreso indirecto que tiene el gran inconveniente de que no se incorpora de manera definitiva al salario base sino que desaparece cada año ${ }^{6} \mathrm{y}$ solo se sostiene si el investigador muestra evidencias de productividad.

El número y monto de las becas varía de institución a institución. La UNAM tiene un Programa Institucional de Apoyo al Desempeño (PRIDE). La UAM posee cinco becas (por grado académico, por docencia, por estímulo a la docencia e investigación, por trayectoria sobresaliente, y de apoyo a la permanencia), el resto de instituciones solo posee la beca al desempeño académico pero internamente definen muchas variantes para la asignación de estas becas.

El salario base de los investigadores apenas cubre el $41 \%$ del ingreso total. La antigüedad llega al 9\% que si los sumamos llega al 50\% del ingreso. Dada la normatividad existente, ésta es la porción que un investigador percibiría en el caso de que llegara a jubilarse y peor le iría si enfermase. Es decir, la jubilación representa para los investigadores una reducción súbita de la mitad de su salario. Dicho de manera grotesca, un investigador no puede enfermarse ni envejecer.

Si un investigador quiere hacer atractivo su salario, deberá sujetarse a todas las normas de participación que existan y se emitan en el futuro. En este momento se debe someter al menos a tres procesos de evaluación (Becas internas que tienen diversos nombres en cada institución, la beca del SNI y aunque no es beca económica PROMEP también evalúa cada tres años). Se debe adicionar las que se hacen a nivel interno en cada institución y que -sin brindar apoyo económico- se hacen periódicamente para conformar los Padrones de investigadores de cada institución, más las tradicionales de los alumnos en cada curso que impartan.

Existe una especie de "limbo" intelectual dentro de las universidades o IES en general que eufemísticamente se conoce como "plazas por honorarios" o "por obra determinada", que son plazas temporales, donde lo más común es que no se reconoce la antigüedad, por más que haya casos en donde algún investigador haya ocupado una plaza de este tipo por más de diez años. La razón de existencia de este limbo intelectual es por la carencia de plazas definitivas que solo se autorizan con crecimientos significativos y consistentes en el presu-

6 Cada institución tiene diferentes maneras de resolver el tema de las becas de productividad. En algunos casos la revisión es de mayor plazo o dejan un fondo de productividad que no se pierde. De la misma manera cada institución tiene diferentes bolsas disponibles para este tipo de becas. 
puesto de las instituciones. Aquí el problema deja ver una cara oculta: la de una carencia de actualización en el tipo de relaciones que deberían practicarse con este segmento laboral que desde el punto de vista de la teoría de los mercados laborales, debía recibir un mejor trato en sus relaciones de trabajo ya que implica a los trabajadores con mayor nivel de formación escolar de todo el mercado.

En general podemos decir que la gama de ingresos de los investigadores es muy amplia y puede oscilar desde 15 mil pesos mensuales en las condiciones más desventajosas, hasta 80 mil pesos para los casos más favorecidos. El salario promedio nacional (muestral) para el 2009, es de 36,789 pesos por mes, lo cual los ubica dentro de una franja de ingresos no alcanzable fácilmente dentro del mercado de trabajo en general en México.

Un investigador reconocido por el SNI puede vivir dignamente y sin grandes apuros económicos en México, mientras no se presente una circunstancia extraordinaria; en cuyo caso su estabilidad sucumbe con facilidad. Es decir, se trata de buenas condiciones de estabilidad laboral y salarial mientras esté en activo y cumpliendo los requisitos exigidos por las diversas instituciones que aportan a su salario global, pero no dejan de contener un límite que le puede llevar -en situaciones críticas nada especiales y casi inevitables (enfermedad, incapacidad o jubilación)- a una condición más o menos precaria.

Los datos de ingreso obtenidos a partir de las entrevistas realizadas nos muestra un mayor beneficio para los investigadores contratados en instituciones donde hay mayor concentración.

\begin{tabular}{|c|c|c|c|}
\hline & Nacional & DF & Provincia \\
\cline { 2 - 4 } Salario promedio de & & & \\
los investigadores & 36,789 & 46,837 & 28,878 \\
\hline
\end{tabular}

Cuadro n. 5: México. Evolución de los salarios mensuales promedio de investigadores de las entidades bajo estudio según área de trabajo

Fuente: Encuesta UAM-FEBUAP (2009). México

Es claro que el primer nivel de diferenciación se encuentra en los salarios, dependiendo de donde se ubica la institución de trabajo. Aquellos investigadores que laboran en centros del DF tienen un ingreso $27 \%$ por arriba del promedio nacional y aquellos que trabajan en provincia ganan en promedio 18 mil pesos 
menos que los del DF y 8 mil pesos menos que el valor de la media en valores aproximados, lo cual en términos porcentuales respecto a la media nacional es un $38 \%$ menos.

\begin{tabular}{|c|c|c|}
\hline & Hombres & Mujeres \\
\cline { 2 - 3 } $\begin{array}{c}\text { Salario promedio } \\
\text { de los investigadores }\end{array}$ & 39,093 & 31,650 \\
\hline
\end{tabular}

Cuadro n. 6: México. Evolución de los salarios mensuales promedio de investigadores de las entidades bajo estudio según sexo.

Fuente: Encuesta UAM-FEBUAP (2009). México

La variable sexo también explica las diferencias en el mercado de trabajo de los investigadores, pues en términos de ingreso, las mujeres ganan menos que los hombres.

En valores absolutos las investigadoras ganan 7, 500 pesos menos al mes que los investigadores y si la comparación la hacemos respecto al salario promedio tenemos que los investigadores ganan -solo por el hecho de ser del sexo masculino- un $6 \%$ arriba del promedio nacional y las investigadoras ganan -solo por el hecho de ser del sexo femenino- un $14 \%$ menos que el promedio nacional.

\begin{tabular}{|c|c|c|}
\hline $\begin{array}{c}\text { Salario promedio de investi- } \\
\text { gadores mujeres }\end{array}$ & DF & Provincia \\
\cline { 2 - 3 } & 41,410 & 25,550 \\
\hline
\end{tabular}

Cuadro n. 7: México. Evolución de los salarios mensuales promedio de investigadoras de las entidades bajo estudio según área de trabajo.

Fuente: Encuesta UAM-FEBUAP (2009). México

Los efectos de la concentración sobre la estructura del salario de los investigadores es muy clara pero también se filtran una serie de efectos derivados de la naturaleza desigual del mercado en el que se ven afectadas de manera doble las investigadoras. 


\subsection{Otros efectos}

Existen otros efectos no asociados a la concentración y no necesariamente negativos, que se desean mostrar como característicos de este segmento de mercado lleno de sorpresas. Se exhibe un comportamiento atípico respecto a la edad, más allá de lo dicho en cuanto a obstaculizar el ingreso de los jóvenes, lo cual no resulta atípico. A lo que nos referimos es a que los ingresos son mayores cuando se acumula mayor experiencia y por ende mayor edad. Según la edad de los investigadores y sus ingresos se podría dibujar un gráfico en forma de media " $U$ ", es decir tiene un inicio con bajos valores de ingreso y van creciendo de manera no claudicante con mayor edad, condición que no se observa en otros mercados.

Otro rasgo atípico de este segmento es el que tiene que ver con la designación de un límite a la vida productiva como trabajador, que a nivel nacional para todo el mercado se ubica aproximadamente a los 40 años de edad. En el caso de los investigadores este límite se extiende hasta los 60 años

\section{CONCLUSIONES}

El mercado de trabajo de los investigadores está en crecimiento, proporciona ingresos por arriba de cualquier promedio a los que participan de él y ciertas condiciones de estabilidad aunque extraordinariamente frágiles. Es un mercado institucional donde sus normas generan una imagen de relativa igualdad.

Es un segmento de mercado que se está feminizando gracias a la capacidad de resiliensia de las investigadoras. La principal fuente de obstáculos de este mercado es su excesiva centralización institucional que impide un mayor crecimiento, una mayor racionalidad a su crecimiento y genera bloqueos a los espacios de los estados con mayor debilidad en la investigación.

Este mercado reproduce desigualdades típicas de cualquier mercado, aunque no esperables en él, donde concurren los buscadores con mayor nivel de formación académica de todo el país. Las desigualdades más evidentes ocurren con las investigadoras y con los investigadores jóvenes.

Una política de desarrollo más racional de este mercado debería incluir políticas de ciencia menos "ciegas", actualmente tienen una orientación universal pero deberán estar más orientadas a fortalecer ciertas regiones geográficas y ciertas instituciones y además, cultivando ciertos temas de investigación de importancia estratégica para nuestro desarrollo como país. 
El mercado de los investigadores puede mantener su crecimiento de manera virtuosa incluyendo generaciones de jóvenes investigadores y de investigadoras haciendo un uso más eficiente de los actuales centros mediante una reestructuración funcional que apoye su crecimiento aplicando los criterios de orientación temática y de aseguramiento de la calidad y eficiencia ya existentes. Se tiene un 30\% de centros de investigación con debilidad numérica, ese debe ser un nicho a estudiar con detenimiento para definir condiciones de elevación de la productividad y esclarecimiento de la pertinencia.

Se debe plantear la necesidad de una segunda "institucionalización" de la investigación en México, en la cual se busquen nuevas condiciones de rendimiento, productividad y pertinencia, pero además nuevas condiciones de desempeño laboral para los investigadores, manteniendo los atractivos de esta actividad laboral pero garantizando una mayor estabilidad, eliminando la actual fragilidad salarial.

\section{REFERENCIAS}

ANUIES. Estadísticas de la educación superior. México: ANUIES, 1999.

ANUIES. Catálogo de posgrado en universidades e institutos tecnológicos.. México: ANUIES, 2006

ARTILES MARTIN, A. Y.; KOHLER, H. Manual de la sociología del trabajo y de las relaciones laborales. Madrid: Delta Publicaciones, 2007.

CAMPOS, G. Informe final del proyecto: El mercado de trabajo de los investigadores en el centro de México. UAM-BUAP-CONACyT. Mimeo, México. 2009.

CONACYT. Listado de investigadores en el SNI. México: CONACYT, 2008.

CONACYT. Listado de investigadores en el SNI. México: CONACYT, 2009.

IZQUIERDO, Isabel.La formación de investigadores y el ejercicio profesional de la investigación: el caso de los ingenieros y físicos de la UAEM. Revista de Educación Superior, México, v. 35, n. 40, p 7-28, oct./ dic. 2006. 
KERR, C. Mercados de trabajo y determinación de los salarios. La "balcanización" de los mercados de trabajo y otros ensayos. España: Ministerio de Trabajo y Seguridad Social, 1954/1985.

LOMNITZ L.; FORTES J. Ideología y socialización. El científico ideal. UNAM. México: IIMAS, 1981.

LOMNITZ, LARIZA. Estructura de organización social de un instituto de investigación. En: LOMNITZ, Larissa Adler. Lo formal y lo informal en las sociedades contemporáneas. Chile: DBAM-CI Diego Barros, 2008. PIORE, M.; DOERINGER, P. Mercados internos de trabajo y análisis laboral. 2. ed. España: Ministerio del Trabajo y Seguridad Social, 1985.

REYGADAS, L. La Apropiación. Destejiendo las redes de la desigualdad. Barcelona: UAM-Antrophos, 2008.

SÁNCHEZ DAZA, G. Directorio CECyT. Centros de Investigación e Instituciones de Educación Superior del Estado de Puebla. Ed. Consejo Estatal de Ciencia y Tecnología. 2000.

UAM-BUAP. Encuesta. En: CAMPOS, G. R. Informe final del proyecto "El mercado de trabajo de los investigadores en el centro de México. UAMBUAP-CONACyT. Mimeo, México, 2009.

WITH, L. Breaking through the glass ceiling. Woman in managment. Revista de la OIT, Ginebra, n. 39, jun. 2001. 